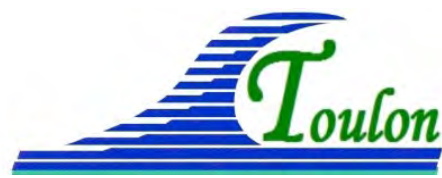

$$
\begin{aligned}
& \text { XIV }{ }^{\text {èmes }} \text { Journées Nationales Génie Côtier - Génie Civil } \\
& \text { Toulon, } 29 \text { juin au } 1^{\text {er }} \text { juillet } 2016
\end{aligned}
$$

DOI:10.5150/jngcgc.2016.015 (C) Editions Paralia CFL

disponible en ligne - http://www.paralia.fr - available online

\title{
Gestion intégrée, risques côtiers et aménagements sur les littoraux coutumiers océaniens français. Les apports croisés de la gestion participative et des méthodes classiques
}

\author{
Michel ALLENBACH ${ }^{1,2}$, Matthieu LE DUFF ${ }^{1,3}$, \\ Pascal DUMAS $^{3}$, Olivier COHEN ${ }^{4}$
}

1. Université de la Nouvelle-Calédonie, LIVE- EA 4243, BP R4 98851, Nouméa,

Nouvelle-Calédonie.michel.allenbach@univ-nc.nc

2. Labex CORAIL.

3. Université de la Nouvelle-Calédonie, CNEP - EA 4242, BP R4 98851, Nouméa, Nouvelle-Calédonie.

4. Université du Littoral de la Côte d’Opale (ULCO), UMR 8187, Dunkerque, France.

\section{Résumé :}

Les littoraux sur zones coutumières des trois provinces néo-calédoniennes et du Territoire des Îles Wallis et Futuna sont soumis à des statuts dérogatoires par rapport aux règles nationales. A partir de quelques exemples, la communication se propose de décrire certains aspects des spécificités des statuts de ces espaces littoraux et les conséquences induites en terme de gestion intégrée, de prévention des risques côtiers et d'aménagements à mettre en place pour réduire les effets de l'érosion et des submersions marines. Sont présentés les différents stades de la création d’observatoires participatifs de l'environnement, instruments d'acquisition de données, de partage et de mise en dialogue des savoirs "habitants" et des savoirs "experts" pour une gestion intégrée de l'espace littoral.

Mots-clés : Gestion intégrée, Espace littoral, Statut, Observatoire participatif, Îles Loyauté, Wallis et Futuna, Pacifique sud-ouest.

Abstract: Coastal areas on customary land of the three New-Caledonian provinces and Territory of the Wallis and Futuna Islands are subjected to derogatory statutes in relation to national rules. Through some examples, the communication aims to describe specific aspects of statute of these coastal areas and the consequences induced in terms of integrated management, prevention of coastal hazards and facilities put in place to reduce the effects of erosion and coastal flooding. Are presented the different stages of the creation of participatory environmental observatories and data acquisition instruments permitting sharing "inhabitants" knowledge and "experts" knowledge for integrated management of the coastal area.

Keys words : Integrated management, Coastal area, Status, participatory Observatory, Loyalty Islands, Wallis and Futuna, Southwest Pacific. 
Thème 2 - Dynamique sédimentaire

\section{Les zones littorales coutumières étudiées}

1.1 Présentation géographique rapide des sites

Les collectivités ultramarines françaises décrites dans la communication sont, d'une part, la Nouvelle-Calédonie et d'autre part, Wallis et Futuna, situées dans le Pacifique sud-ouest (Figure 1).

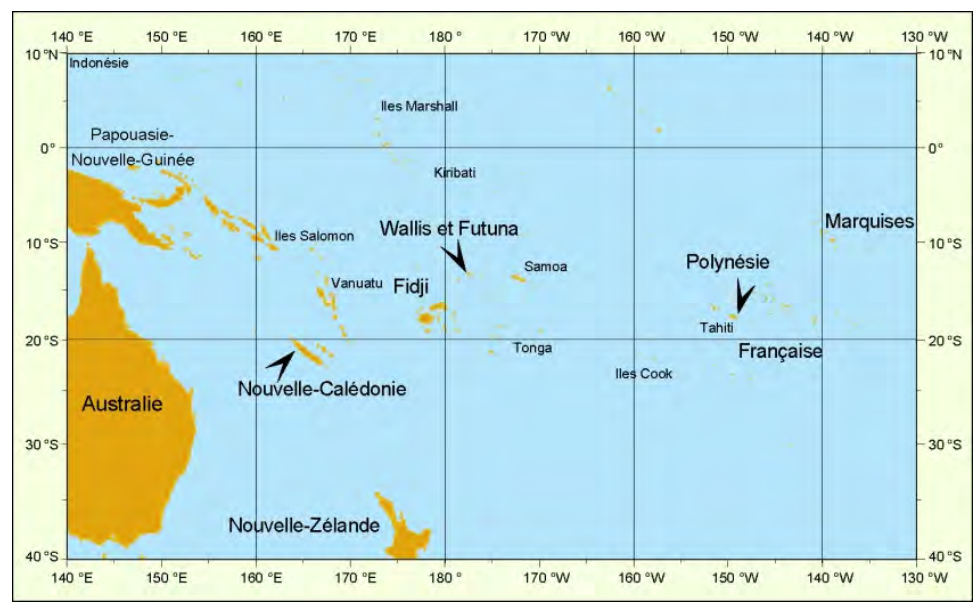

Figure 1. Le sud-ouest Pacifique. Présentation régionale.

En Nouvelle-Calédonie, deux sites sont décrits : l'île d'Ouvea (Province des îles Loyauté) qui fait l'objet de l'essentiel des développements (Figure 2) et l'île Ouen (Province Sud) dont le cas sera brièvement évoqué. A Wallis et Futuna, ce sont les deux îles principales, Uvea sur Wallis et Futuna dans l'archipel de Horn qui seront, elles aussi, rapidement traitées (cas très voisins de celui d’Ouvéa).

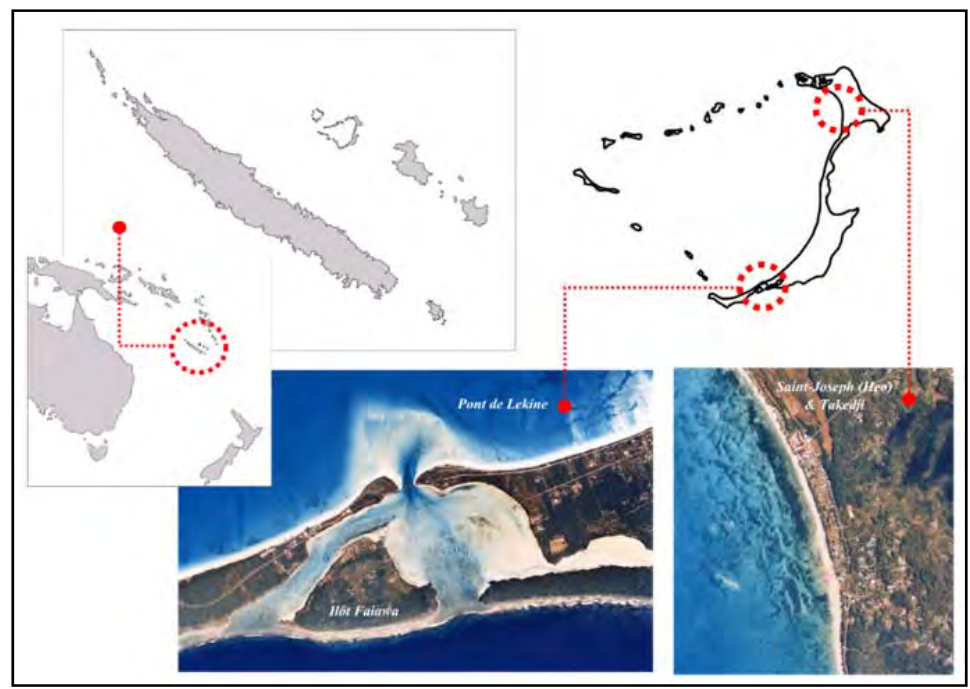

Figure 2. Localisation d'Ouvéa au sein du Pacifique et des chantiers des sites de SaintJoseph/Takedji et Lekine. 


\section{XIV ${ }^{\text {èmes }}$ Journées Nationales Génie Côtier - Génie Civil \\ Toulon, 29 juin au $1^{\text {er }}$ juillet 2016}

\subsection{Des statuts politiques spécifiques et une vision océanienne voisine}

\subsubsection{La Nouvelle-Calédonie}

La Nouvelle-Calédonie est une collectivité engagée dans un processus de décolonisation qui est aujourd'hui cadré par la loi organique n 99-209 du 19 mars 1999 mettant en œuvre l'Accord de Nouméa (1998). Les décrets d'application de cette loi ont transféré aux trois provinces néo-calédoniennes la compétence en matière de gestion de l'environnement dans les eaux intérieures et territoriales entourant la Grande-Terre et l'archipel des Loyauté. Les codes de l'Environnement sont aujourd'hui à des niveaux d'élaboration et d'application différents selon les provinces.

La compétence en matière de sécurité civile vient d’être récemment transférée (janvier 2014) à la Nouvelle-Calédonie et sa déclinaison à l'espace littoral, et tout particulièrement aux risques de submersion, est à l'état d'ébauche. Elle se limite actuellement à la mise en place d'un dispositif d'alerte automatisé : les sirènes. Les autorités compétentes sont le gouvernement, les provinces et les communes de Nouvelle-Calédonie mais le dispositif ignore, pour l’instant, une réalité importante, le fait que très tôt, dans l'organisation politique du territoire, les différents statuts successifs mis en place ont reconnu et confirmé l'existence de terres coutumières, par place sur la Grande Terre et générales sur l'Archipel des Loyauté (Arrêté du gouverneur du 22 janvier 1868 sur la propriété indigène). La gestion du foncier de ces terres réputées incessibles, incommutables, insaisissables et inaliénables (la règle des 4 i) et du domaine marin qui leur est associé ( $c f$. paragraphe 1.2.3) est réalisée par les clans propriétaires sur la base de règles non écrites transmises par l'oralité. Il s'en suit une surimposition de compétences et de visions différentes de l'espace littoral qui rendent complexes les actions de prévention des risques littoraux et la réalisation d’aménagements coordonnés.

\subsubsection{Wallis et Futuna}

Les îles de Wallis et Futuna ont été rattachées administrativement à la République française par référendum en 1959, date d'élaboration d'un statut politique qui les distinguent de toutes les autres collectivités ultra-marines françaises. Ce statut juxtapose, en effet, aux pouvoirs républicains exécutif (État) et législatif (Assemblée territoriale), un pouvoir coutumier fort (2 royaumes à Futuna et un à Wallis). Le statut reconnaît le double respect à la religion catholique et à la coutume. Les terres sont également incessibles et inaliénables, mais contrairement à la Nouvelle-Calédonie où il subsiste les restes plus ou moins affirmés d'un domaine public maritime hérité d'une époque où l'Etat transposait aux collectivités ultra-marines du Pacifique l'essentiel de la législation métropolitaine, cette notion n'existe pas sur Wallis et Futuna. Chaque famille propriétaire d’un terrain sur le littoral l'est également sur l'espace maritime situé au droit de ce terrain et aucun conflit exprimé n’a pu esquisser la moindre jurisprudence en 


\section{Thème 2 - Dynamique sédimentaire}

la matière. Tout reste donc à faire sur ce territoire pour mettre en place les outils juridiques permettant de gérer un intérêt collectif, qu'il faudrait d'ailleurs auparavant définir avec les habitants et les chefferies. Un code de l'environnement qui prend en compte l'espace littoral a été écrit, mais il n'est pas appliqué car non accepté par les chefferies et la population. Les risques de submersion, majeurs sur Futuna, du fait du caractère sismique de la zone et de l'absence de récif-barrière, sont pris en compte par le biais d'un réseau d'alerte automatisé de sirènes. Comme en Nouvelle-Calédonie, sur Wallis et Futuna, se surimposent des visions différentes de l'espace littoral entre techniciens, le plus souvent expatriés, et populations locales qui rendent obligatoires l'intégration de la vision océanienne de l'espace littoral transmise par la coutume si l'on veut être efficient en termes d'aménagements littoraux et de prévention des risques.

\subsubsection{Coutume et vision océanienne de l'espace littoral}

Le préalable à une gestion efficace de l'espace littoral est une connaissance approfondie de son fonctionnement et de son évolution du point de vue tant morpho-dynamique (JEANSON et al., 2010) que socio-culturel. Cette affirmation prend toute sa signification en Océanie. En effet, si la compréhension morpho-dynamique est une entrée classique des problématiques littorales, le fonctionnement socio-culturel sur les espaces géographiques concernés par la communication est fondamentalement différent de celui connu en métropole ou même dans les départements d'outre-mer. Il s'appuie sur la coutume, un ensemble de règles non écrites qui détermine les relations sociales et régit tous les actes au quotidien des populations et c'est à travers le filtre de la coutume qu'il faut concevoir les actions à mener sur le littoral, afin qu'elles soient comprises et acceptées par la population. Il est essentiel, à ce niveau, de prendre en compte la vision océanienne de l'espace littoral qui veut que chacun soit maître devant chez soi. Cette règle s'impose face à l'intérêt collectif qui ne peut prévaloir qu'à travers la recherche d'un consensus, toujours précaire. Il est donc malaisé de réaliser des aménagements intégrés qui satisfassent toutes les parties, et à l'inverse, de s'opposer à une initiative individuelle, même si elle est source de dysfonctionnements pour les segments littoraux adjacents au lieu où elle est réalisée.

\subsubsection{Coutume et attachement à la terre}

L'attachement à la terre, leur terre, est un dénominateur commun du mode de vie des peuples océaniens, mais c'est en Nouvelle-Calédonie qu'il s'exprime de la façon la plus affirmée sur l'espace ultramarin français du Pacifique et c'est là que nous le commenterons. Cet attachement doit être considéré à la lumière de 3 facteurs principaux : culturel, politique et historique. Nous évoquerons ici rapidement un seul d'entre-eux, le facteur culturel qui peut se transposer en partie au cas des Îles Wallis et Futuna. L'homme kanak se définit avant tout par sa relation avec la terre, c'est une question identitaire. Le lien à la terre traduit la relation affective liant la famille/ le clan 


\section{XIV èmes Journées Nationales Génie Côtier - Génie Civil \\ Toulon, 29 juin au $1^{\text {er }}$ juillet 2016}

et la terre. Lui retirer sa terre, c'est lui enlever son nom, le priver de ses relations sociales, de sa place et sa fonction dans la coutume, c'est au final lui retirer ce qui le définit en tant qu'homme au sein de sa société. Sur cette base, il est impossible d'envisager la transposition directe du modèle métropolitain de gestion du risque littoral. L'expropriation est impossible et le déplacement des populations ne peut se faire que par consensus inter clanique.

\section{La démarche des observatoires participatifs appliquée aux spécificités océaniennes : l'exemple de l'île d'Ouvéa}

\subsection{Le contexte face au risque de submersion}

Une récente étude relative au risque tsunami (LE DUFF, 2013) souligne qu'à Ouvéa, le long de la façade Ouest de l'île, au sein d'une bande côtière large de 500m à compter de la limite du trait de côte et où les altitudes restent inférieures à 10m, on dénombre la présence de :

- 40\% de la population résidente ;

- 50\% des Installations Classées pour la Protection de l'Environnement (ICPE) ;

- 50\% des Etablissements Recevant du Public (ERP) ;

- $80 \%$ des structures touristiques.

C'est donc à la fois l'intégrité physique d'une large partie de la population qui est directement soumise à l'influence des forçages météo-marins, mais également l'ensemble de l'architecture économique et administrative de l'île. L'exposition des réseaux : routiers, téléphoniques et électriques, qui jalonnent ces espaces doit également être prise en considération dans l'évaluation globale de la vulnérabilité de l'île aux risques côtiers. A ce titre, Ouvéa présente un degré de vulnérabilité important, que l’on considère la question des forçages météo-marins, submersions marines liées aux passages de cyclones, coups d'Ouest, tsunamis ou encore les conséquences à venir de l'élévation du niveau de la mer en lien avec le réchauffement climatique.

Le questionnement est de savoir comment prévenir les risques sur un littoral géré par les autorités coutumières et sur lequel la relation entre le risque et le territoire est fondamentale. Le lien à la terre chez les kanak revêt une dimension identitaire profonde, où tout acte de migration spatiale et d'implantation dans un espace nouveau s'inscrit dans un réseau complexe de liens sociaux et de représentations symboliques. La terre est l'objet de tous les enjeux et fixe les rivalités et les conflits entre les groupes. Il convient d'intégrer cette réalité dans les solutions à proposer quant à la stratégie à adopter en matière de gestion de ce risque.

\subsection{Le contexte face à l'érosion des sites}

Comme c’est le cas sur l'essentiel des plages des îles et îlots néo-calédoniens, le littoral d'Ouvéa est en déflation. Dans le cadre d'un programme européen (INTEGRE) et d'un 


\section{Thème 2 - Dynamique sédimentaire}

programme national (MOM "Litto Ouvea"), deux chantiers géographiques sont suivis avec pour objectifs la compréhension de l'évolution géomorphologique des sites et la définition de solutions pour faire face aux tendances érosives constatées. Le premier (2 km de long) est situé au nord de l'île, sur la côte des tribus de Saint-Joseph (Heo) et Takedji. Il s'agit d'une côte sableuse lagonaire rectiligne orientée nord-sud. Le second qui s'étend sur un linéaire d'environ $4 \mathrm{~km}$ est situé au sud de l'île et il encadre le pont de Lekine. Ce dernier surplombe le chenal marquant la limite entre le lagon d'Ouvéa et un petit lagon intérieur dit "lagon de Faiawa". Deux pointes sableuses en vis à vis encadrent le chenal. Sur ces deux secteurs, les actions anthropiques (polders, épis, pont) génèrent l'essentiel de l'érosion observée.

La perte d'espace, par les processus érosifs, n'a pas le même impact sur les groupes composant la société locale en fonction de la valeur que chacun d'entre eux attribue aux lieux. Cette valeur peut être symbolique, c'est à dire rattachée à un système de représentations en lien avec l'identité ou l'histoire d'un clan ; économique si le lieu est exploité au niveau touristique ou agricole, mais également patrimoniale. Pour les populations, largement influencées par les médias, la perturbation de leur environnement (érosion) est souvent associée aux conséquences du réchauffement climatique. Une mauvaise interprétation des mécanismes en jeux entrainera toute une série de comportements débouchant sur des erreurs de gestion qui aggraveront une situation potentiellement délicate.

\subsection{L’intérêt des observatoires participatifs}

Les éléments contextuels présentés dans les paragraphes précédents rendent nécessaire une véritable adaptation de la démarche "experte" dans une dynamique participative impliquant les "habitants". Ils sous-tendent, en effet, l’intégration de facteurs qualitatifs d'ordre culturel dans l'évaluation des processus affectant les zones littorales. Ces facteurs sont directement liés à la valeur attribuée à un espace donné par un ou des groupes composant la société concernée. Ces éléments doivent donc être connus et compris par les intervenants et techniciens extérieurs, pour qu'un réel échange puisse s’opérer avec les "habitants" et que les savoirs qui seront échangés puissent être compris dans leur dimension socio-territoriale. Les observatoires participatifs sont la méthode proposée pour favoriser ces échanges et permettre des prises de décision efficientes. Le point d'accroche de ce réseau participatif est l'observation du rivage, et notamment de l'évolution du trait de côte, au regard des dynamiques érosives. Compte tenu de la menace directe aux biens (les maisons construites sur la dune littorale attaquée par l'érosion), l'observatoire participatif constitue une étape cruciale pour permettre aux populations et à l'autorité coutumière, premier acteur de la gestion du littoral sur nos terrains d'études, d'acquérir certaines compétences et d'approfondir leurs connaissances sur ce milieu. Au-delà de l'érosion, il est possible par cette approche d'évoquer d'éventuels déplacements de populations vers des zones sécurisées face au risque 


\section{XIV ${ }^{\text {èmes }}$ Journées Nationales Génie Côtier - Génie Civil \\ Toulon, 29 juin au $1^{\text {er }}$ juillet 2016}

tsunami notamment, de limiter les implantations d'infrastructures dans les zones menacées et d'envisager la destruction des ouvrages qui perturbent le fonctionnement hydrosédimentaire des sites.

\subsection{La mise en œuvre de l'observatoire participatif d'Ouvea}

Elle s'inscrit dans la logique partenariale entres "savoirs experts" et "savoirs locaux". La mairie, la province des îles, le GDPL (Groupement de Droit Particulier Local) "Bowene Tapu", l'Association pour la Sauvegarde de la Biodiversité d'Ouvéa (ASBO), ainsi que différents guides de randonnées se sont regroupés au sein d'un comité de réflexion portant sur les pressions s'exerçant sur le littoral de l'île et notamment sur celui des tribus de Saint-Joseph et Takedji où les processus d'érosion côtière inquiètent particulièrement la population. L’Université de la Nouvelle-Calédonie a intégré ce groupe de réflexion et proposé la mise en place d'un suivi de l'érosion dans le cadre d'un observatoire participatif sur financement croisé du Gouvernement de la NouvelleCalédonie, de l'Europe (Programme INTEGRE) et du Ministère des Outremers (Programme MOM).

Le travail mené par l'Université est classique. L'évolution géomorphologique des sites est tout d'abord étudiée à une échelle spatiale et temporelle relativement large, s'appuyant sur l'analyse diachronique (60 ans), d'images aériennes et satellitaires de l'île d'Ouvéa. Parallèlement, un suivi des processus de dynamique sédimentaire littorale actuels affectant les littoraux sableux d'Ouvéa est réalisé. Il s'agit de mesurer les tendances évolutives du littoral à des échelles spatiales et temporelles beaucoup plus fines. Le travail est mené sur les plages de Saint Joseph et de Mouli qui sont attaquées de façon significative par des processus érosifs, sur la base de levés répétitifs au fil du temps sur une période de deux ans. Les mesures de topométrie sont réalisées par le biais d'un arsenal méthodologique très diversifié mixant des méthodes très précises (DGPS, photo restitution à partir de levés aériens par drone), mais onéreuses et des méthodes moins sophistiquées (levé au cadre, niveau de chantier), d’un degré de précision moindre, mais beaucoup moins coûteuses et d'un usage réclamant moins de technicité pour pouvoir être utilisées en routine par les populations locales, partie prenante de la démarche. La finalité est ici, dans un espace océanien démuni de ressources, de trouver les moyens de suivre les faits affectant les petites îles isolées, d'être réactifs aux phénomènes climatiques qui viennent impacter les littoraux, mais surtout de pouvoir envisager l'acquisition de données pérennes lorsque les financements sur projets, de durée limitée, arrivent à leur terme. La formation d'observateurs tout au long de l'étude, couplée à la réalisation de larges campagnes d'informations/sensibilisations et l'entretien d'un lien solide avec les structures administratives locales et les représentants coutumiers constituent les éléments indispensables à la réussite, sur le long terme, d'un tel projet en Océanie. Son intérêt est de premier ordre sur des espaces fonciers où les règlementations en terme d'aménagement et de gestion du territoire sont inexistantes car 


\section{Thème 2 - Dynamique sédimentaire}

relevant quasi-uniquement des autorités coutumières. Plus que n'importe où ailleurs, il est fondamental et incontournable d'associer les populations à la mise en place de ces stratégies, la reconnaissance du droit coutumier en Nouvelle-Calédonie interdisant de fait toute action "centralisatrice" dans les modes de gestion.

\subsection{Les résultats}

La communication n’a pas vocation à présenter les résultats des mesures classiques (levés DGPS et drones, analyse diachronique de l'imagerie) qui ont été exposés lors d'un colloque international qui s'est tenu aux îles Fiji en novembre 2015, par le bais de plusieurs communications (COHEN et al., 2015). Nous souhaitons simplement ici insister sur l'excellente corrélation (Figure 3) obtenue entre les résultats des méthodes sophistiquées et des méthodes simplifiées à travers l'exemple d'un simple profil de plage levé par DGPS et au cadre, selon la méthode d'EMERY (1961). Les variations sont d'ordre centimétrique et les écarts enregistrés tout à fait admissibles pour des interprétations de dynamique sédimentaire littorale.

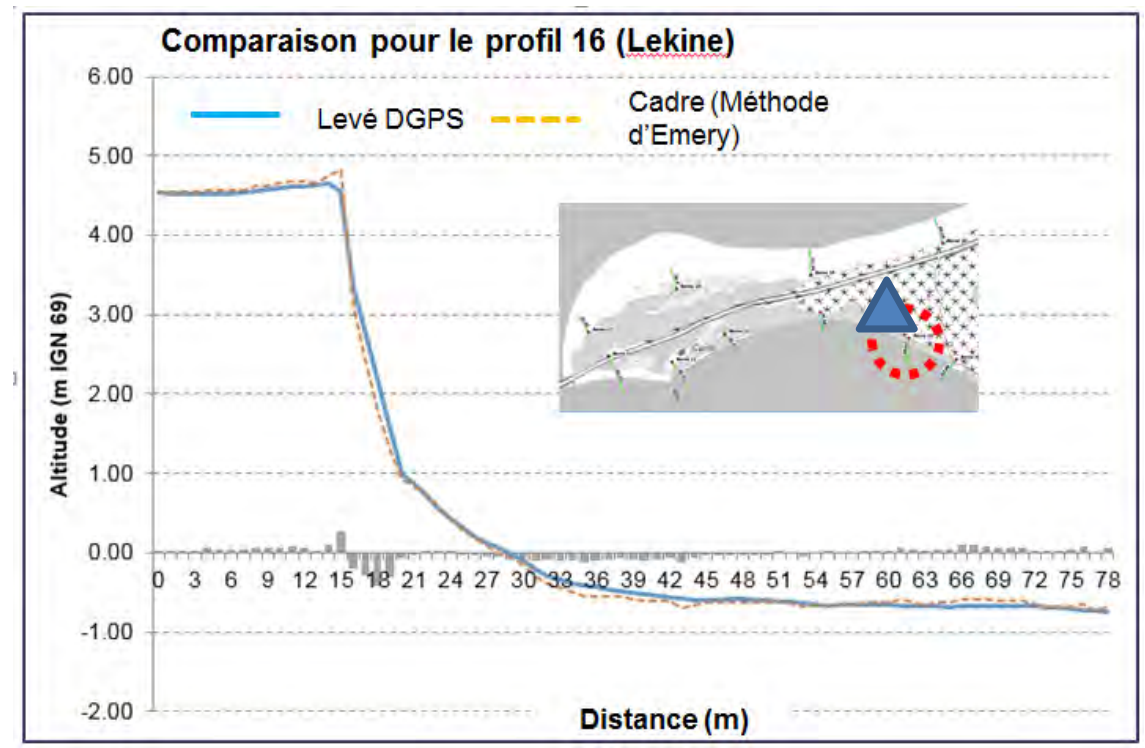

Figure 3. Profil de plage. Comparaison DGPS et cadre d'Emery.

\subsection{La transposition de l'observatoire participatif d'Ouvea à d'autres sites océaniens}

La démarche mise en œuvre à Ouvéa a intéressé la tribu de Ouara sur l'île Ouen en Province Sud. Cette tribu est également éligible au financement INTEGRE dans le cadre des plans de gestion des sites classés au patrimoine mondial et avec le partenariat provincial que cela impose, elle a souhaité mettre en place un observatoire du même type que celui développé à Ouvéa. Les premiers contacts et expertises en ce sens sont programmés au début du mois de mars 2016. Il apparaît donc que la méthode puisse être 


\section{XIV ${ }^{\text {èmes }}$ Journées Nationales Génie Côtier - Génie Civil \\ Toulon, 29 juin au $1^{\text {er }}$ juillet 2016}

étendue à d'autres sites et que les populations autochtones de Nouvelle-Calédonie adhèrent à son esprit.

La démarche est également en cours d'analyse sur le Territoire de Wallis et Futuna où les problèmes d'aménagements littoraux sont complexes (ALLENBACH \& HOIBIAN, 2008) et ne peuvent pas être résolus par le seul recours à l'expertise classique de génie civil, alors même que des fonds importants sont attribués au Territoire pour mettre en place des défenses contre l'érosion. La gestion des secteurs à aménager est soumise aux mêmes contraintes que sur les Îles Loyauté et les contacts entre Loyaltiens et Wallisiens ont amené la réflexion au niveau des décideurs coutumiers et administratifs avec de bonnes chances de voir bientôt la création des observatoires participatifs sur les sites reconnus comme sensibles.

\section{Conclusion}

Face à l'immobilisme que génèrent les relations souvent difficiles entre des pratiques expertes et le ressenti par les populations locales d'Océanie qui ne les comprennent pas et qui ont la possibilité, du fait des statuts politiques qui les régissent, de s'opposer efficacement à leur mise en œuvre, la création d'observatoires participatifs permet l'échange et une meilleure compréhension réciproque pour une gestion plus efficiente de l'espace littoral. En l'état d'avancement des programmes, la démarche est acceptée et les obligations de chacun sont remplies. La pérennité de ces observatoires reste à évaluer lorsque les scientifiques prendront de la distance avec les acteurs locaux. Mais, la prégnance des phénomènes érosifs qui touchent de plein fouet des sites littoraux habités de longue date par les populations devrait les amener, sous le contrôle des autorités coutumières, à pérenniser les observations pour une meilleure connaissance des faits physiques qui touchent ces espaces et ce, afin de pouvoir prendre les décisions socio culturellement acceptables face aux risques.

\section{Références bibliographiques}

ALLENBACH M., HOIBIAN T. (2008). Enjeux, spécificités et perspectives de la gestion intégrée du littoral dans l'espace insulaire français du sud-ouest Pacifique. Européan Journal of Environmental and civil Engineering, Vol. 12(1-2), pp 15-34. http://dx.doi.org/10.1080/19648189.2008.9692993

COHEN O., DUMAS P., LE DUFF M., ALLENBACH M. (2015). Using UAV and very high resolution photogrammetry to assess shoreline evolution. Example in Ouvea, Loyalty Island, New-Caledonia. GIS \& RS Conference, Suva, Fiji Islands from 16-20 novembre 2015.

EMERY K.O. (1961). A simple method of measuring beach profiles. Limnology and oceanography, Vol. 6, pp 90-93. http://dx.doi.org/10.4319/lo.1961.6.1.0090 
Thème 2 - Dynamique sédimentaire

JEANSON M., DOLIQUE F., ANTHONY E.J. (2010). Un réseau de surveillance des littoraux face au changement climatique en milieu insulaire tropical : l'exemple de Mayotte. VertigO, Vol. 10, n³, 19 p.

LE DUFF M. (2013). Rapport de synthèse : Prévention du risque tsunami aux îles Loyauté. Rapport administratif (Subdivision Administrative des Iles Loyauté), Lifou. 